\title{
Electron cyclotron current drive and suprathermal electron dynamics in the TCV tokamak
}

\author{
S. Coda, S. Alberti, P. Blanchard ${ }^{\mathrm{a}}$, T.P. Goodman, \\ M.A. Henderson, P. Nikkola, Y. Peysson ${ }^{1}$ and O. Sauter
}

Centre de Recherches en Physique des Plasmas, Association EURATOM-Confédération Suisse, Ecole Polytechnique Fédérale de Lausanne, CRPP - EPFL, CH-1015 Lausanne, Switzerland

${ }^{1}$ Département de Recherches sur la Fusion Contrôlée, Association EURATOM-CEA, CEA/Cadarache, 13108 Saint Paul-lez-Durance Cédex, France

E-mail: stefano.coda@epfl.ch

Received 15 November 2002, accepted for publication 6 October 2003

Published 3 November 2003

Online at stacks.iop.org/NF/43/1361

\begin{abstract}
Electron cyclotron current drive (ECCD) is an important prospective tool for tailoring the current profile in nextstep devices. To fill the remaining gaps between ECCD theory and experiment, especially in the efficiency and localization of current drive, a better understanding of the physics of suprathermal electrons appears necessary. In $\mathrm{TCV}$, the fast electron population is diagnosed by a multichordal, spectrometric hard x-ray camera and by a highfield side electron cyclotron emission radiometer. The main modelling tool is the quasilinear Fokker-Planck code CQL3D, which is equipped with a radial particle transport model. Systematic studies of fast electron dynamics have been performed in TCV with modulated or pulsed electron cyclotron power, followed by coherent averaging, in order to identify the roles of collisional relaxation and radial transport in the dynamics of the suprathermal population. A consistent picture is emerging from experiment and modelling, pointing to the crucial role of the radial transport of suprathermal electrons in the physics of ECCD.
\end{abstract}

PACS numbers: 52.55.Fa, 52.55.Wq, 52.35. Hr

\section{Introduction}

Electron cyclotron current drive (ECCD) is an important tool for current profile shaping in magnetically confined plasmas, thanks to the highly localized power deposition of the EC wave and the ease of external control of its deposition location and wave-number spectrum $[1,2]$. The strong influence exerted in turn by the current profile shape on the stability of the plasma to MHD modes, as well as on its confinement properties, motivates much current ECCD research. In experimentally relevant plasma conditions, the EC waves propagate from vacuum or air to the resonance layer in the plasma without encountering any cutoff, allowing the launching structures to be placed far from the harsh plasma environment, as may be necessary in a reactor.

The development of high-power, high-frequency, longpulse gyrotron sources in recent years has provided the fusion

a Present and permanent address: Département de Recherches sur la Fusion Contrôlée, Association EURATOM-CEA, CEA/Cadarache, 13108 Saint Paul-lez-Durance Cédex, France. community with a versatile and mature technology for nextstep thermonuclear fusion devices [3,4]. The TCV tokamak $\left(R=0.88 \mathrm{~cm}, a=0.25 \mathrm{~cm}, I_{\mathrm{p}} \leqslant 1 \mathrm{MA}, B_{\phi} \leqslant 1.54 \mathrm{~T}\right)$ is equipped with a $4.5 \mathrm{MW}$ EC heating system, powered by six second harmonic $(\mathrm{X} 2,82.7 \mathrm{GHz})$ and three third harmonic (X3, $118 \mathrm{GHz}$ ) 0.5 MW gyrotrons. An extremely flexible EC beam delivery system, allowing real-time poloidal and toroidal steering, matches the equally flexible plasma position and shape control system of TCV [5].

ECCD experiments have been performed in TCV with the X2 system, for a total delivered power of up to $2.8 \mathrm{MW}$, in a wide variety of plasma shapes, with a broad range of parallel wave numbers and heating locations. In particular, steady-state, fully non-inductive discharges sustained entirely by ECCD and bootstrap current are routinely obtained in TCV [6-8].

The high-energy electron population created by ECCD is diagnosed primarily with a hard x-ray (HXR) pinhole camera, on loan from Tore Supra, and with a high-field side electron cyclotron emission (ECE) system. HXR bremsstrahlung 
emission is detected by a linear array of CdTe detectors [9] along 14 chords that cover the outboard half of the crosssection. The radial resolution is approximately $2 \mathrm{~cm}$ on the midplane and the energy resolution is approximately $7 \mathrm{keV}$ [10]. Spectral pulse height analysis is performed, and eight energy bins are available for each chord with adjustable thresholds within the $10-200 \mathrm{keV}$ range.

The second harmonic X-mode ECE radiometer observes the plasma along one of three possible horizontal viewlines, two on the high-field side and one on the low-field side, and operates in the $78-114 \mathrm{GHz}$ range with 24 channels of $0.75 \mathrm{GHz}$ bandwidth [11]. The EC radiation observed on the high-field side is dominated by relativistically downshifted emission by the high-energy end of the electron distribution function and can thus be employed to diagnose the suprathermal population [12]. The results discussed in this paper were obtained exclusively with the high-field side viewlines.

The quasilinear Fokker-Planck code CQL3D [13] is employed to model the dynamics of the electron distribution function. The code is coupled to the TORAY-GA ray-tracing module [14] and solves the Fokker-Planck equation in two velocity and one spatial dimensions. The equation includes a quasilinear EC wave damping term, a relativistic collision operator and a model for radial diffusion, with an optional dependence on the parallel velocity, a radial dependence and a particle-conserving advection term.

A concerted application of these diagnostic and numerical tools in a variety of experimental scenarios involving ECCD has generated significant evidence that cross-field transport of suprathermal electrons plays a fundamental role in ECCD physics in TCV, as will be discussed in the remainder of this paper. An initial discrepancy between the predicted and measured ECCD efficiencies has been resolved by the introduction in CQL3D of a suprathermal electron diffusion coefficient of the order of the thermal diffusivity [15]. This also brings the predicted HXR emission considerably closer to the experimental measurements than in the absence of diffusion, although the agreement is not yet satisfactory. Finally, direct experimental evidence of radial transport is obtained from time-resolved HXR and ECE measurements in studies with modulated and pulsed ECCD.

\section{ECCD and suprathermal electrons}

Current drive by electron cyclotron waves is theoretically predicted to operate on electrons travelling at substantially suprathermal velocities [16,17]. Experimental measurements in TCV have confirmed that ECCD is accompanied by the creation and sustainment of a significant suprathermal population. Unmistakable suprathermal features are observed when the EC toroidal injection angle, $\Phi$, is larger than $\sim 10-12^{\circ}$, i.e. when the parallel wave number is finite, as required for ECCD: the HXR spectral distribution deviates from that of a Maxwellian plasma, and both the HXR photon temperature (calculated from an exponential fit to the highenergy component of the spectrum) and the ECE radiative temperature are well in excess of the bulk plasma temperature measured by Thomson scattering $[11,18,19]$. By contrast, with $\Phi \simeq 0$ (pure heating mode) the three temperatures coincide.
(In this paper $\Phi$ is defined as the angle between the EC beam and its projection on the poloidal plane at the launcher, and is positive for co-ECCD.)

The dependence of the suprathermal population on the toroidal injection angle is clearly demonstrated by figure 1 , which shows the effect of sweeping $\Phi$ during a plasma discharge on two out of three launchers: while the bulk temperature and the lower energy HXR signal remain constant, the high-energy HXR emission and the ECE radiative temperature increase rapidly with $\Phi[19]$. This example also illustrates the high degree of external control of multiple deposition locations and toroidal injection angles available on TCV, which has proven instrumental in the application of ECCD to current and pressure profile tailoring [5-7, 20-22].

The presence of a suprathermal electron population created by X2 ECCD in TCV has also been shown to engender enhanced absorption of the $\mathrm{X} 3$ waves over that expected for a Maxwellian plasma [23].

As figure 1 shows, the energy resolved HXR measurement is a particularly sensitive indicator of the relative dynamics of the thermal and suprathermal components. On the other hand, photon statistics in this spectral range tend to limit the intrinsic temporal resolution of the measurement [9]. The ECE diagnostic, by contrast, offers the advantage of good time resolution. However, the interpretation of ECE data is complicated by the fact that a given emission frequency

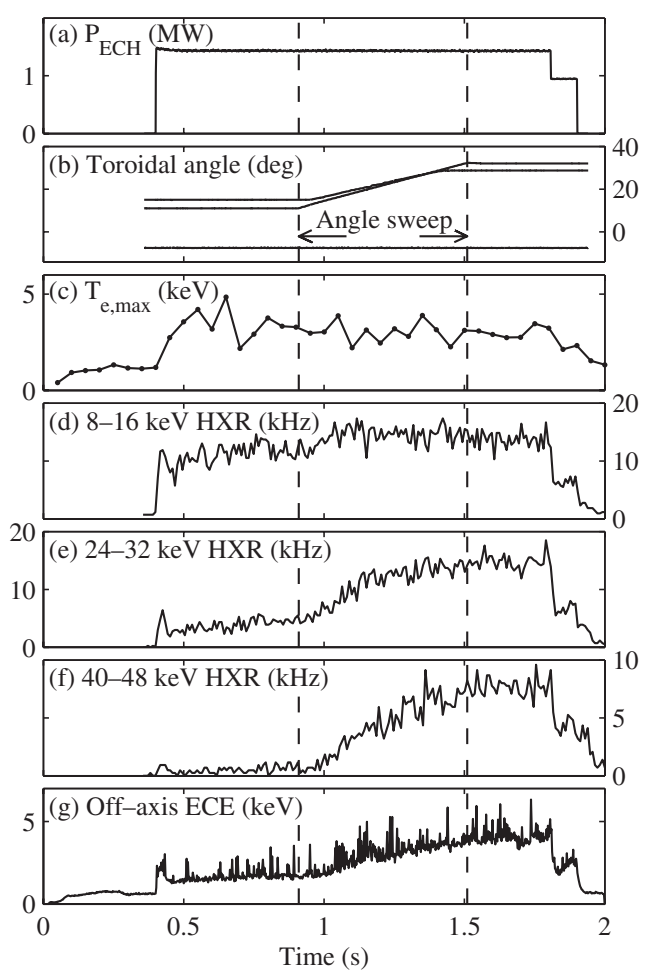

Figure 1. TCV discharge 22035 (limiter configuration, plasma current $I_{\mathrm{p}}=310 \mathrm{kA}$, line-averaged density $\bar{n}_{\mathrm{e}}=1.5 \times 10^{19} \mathrm{~cm}^{-3}$, edge elongation $\kappa_{\text {edge }}=1.4$ ), featuring a sweep of the EC toroidal injection angle on two out of three launchers (from $+11^{\circ}$ to $+32^{\circ}$ and from $+15^{\circ}$ to $+29^{\circ}$, respectively): (a) total EC power, $(b)$ toroidal angles of the three launchers (positive for co-ECCD), $(c)$ peak electron temperature, $(d)-(f)$ three energy channels of the HXR

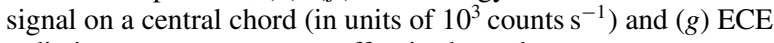
radiative temperature on an off-axis channel. 
ECCD and suprathermal electron dynamics in the TCV tokamak

corresponds to a continuum of spatial positions and electron energies, since the EC frequency depends, relativistically, on both magnetic field and energy. To deconvolve the two quantities some constraints must be applied. The approach that has shown the most promise is based on the assumption of a bi-Maxwellian electron distribution function, describing the bulk and suprathermal populations. This assumption is qualitatively supported by both HXR data and CQL3D modelling. By then applying constraints on the profile shapes for the suprathermal density and temperature, the absolute values of these quantities can be derived from the ECE data [11].

\section{Suprathermal electron dynamics: the role of spatial transport in ECCD}

The physical underpinnings of electron cyclotron resonance heating (ECRH), particularly the power absorption and the power deposition profile, have been validated by numerous experimental measurements [24,25]. Predictions for current drive have also been tested experimentally, with less uniform results: while good agreement with Fokker-Planck quasilinear theory is obtained in some devices [26], discrepancies remain in other cases. In particular, ECCD efficiency in TCV has been generally grossly overestimated by quasilinear theory, which predicted strong non-linear enhancement by the unusually large EC power densities achieved [15]. On the other hand, the total driven current is underestimated by factors ranging from 1 to 3 [27] by linear calculations performed by TORAY-GA, based on the Cohen model [28], which neglects non-thermal effects as well as the current excited in the thermal portion of the electron distribution by electron-electron collisions.

The key to resolving these discrepancies must lie in the dynamics of the suprathermal electron population that governs the physics of ECCD. In particular, cross-field transport of the high-energy current carriers would tend to reduce their local density and correspondingly limit the non-linear enhancement of the current drive efficiency. In TCV we have studied the suprathermal electron dynamics both by direct experimental measurements and by numerical simulations, culminating in crucial comparisons between the two.

\subsection{Experimental measurements}

The spatially resolved, line-integrated HXR measurement can be inverted by various techniques to provide a measure of the local emissivity under the assumption of poloidal uniformity. A systematic study of the inverted profiles in different ECCD aiming geometries and plasma conditions reveals a complex phenomenology that is beyond the scope of this paper. Here we shall focus on two nearly universal observations for toroidal injection angles larger than $20^{\circ}$, which have strong implications for the topic under discussion. First, with predominantly central deposition the inverted profiles are broader than the theoretical power deposition profile calculated by either TORAY-GA or CQL3D (which are invariably in good agreement). Second, with predominantly off-axis deposition, up to $\rho=0.5$, the inverted profiles are centrally peaked. (In this paper $\rho$ is a normalized flux-surface coordinate proportional to the square root of the enclosed volume.)

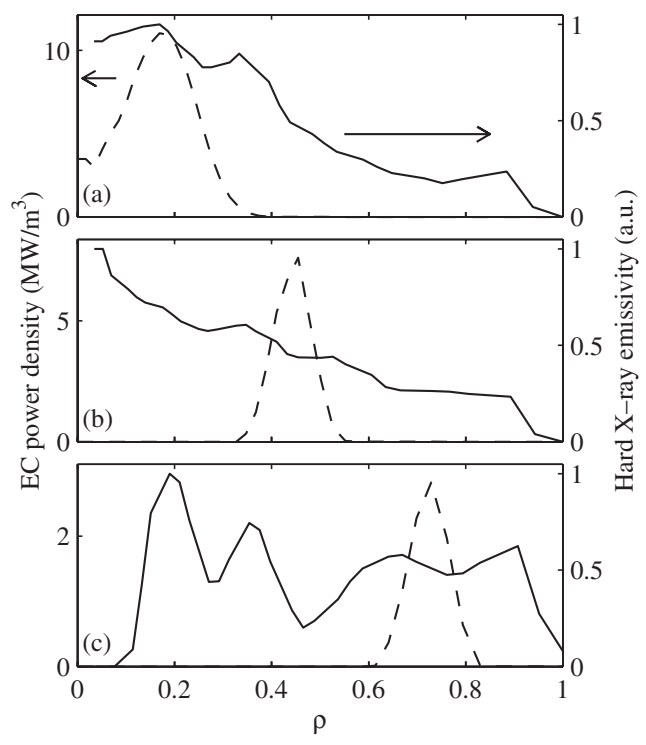

Figure 2. TCV discharges (a) 21982, (b) 21991 and (c) 22003: emissivity profiles of HXRs in the energy range $40-50 \mathrm{keV}(-)$, derived with the Fisher regularization method from the line-integrated profiles averaged from 0.67 to $1.12 \mathrm{~s}$; EC power density profiles (- - - ) calculated by ray tracing. The total injected power is $1.15 \mathrm{MW}$, with $100 \%$ calculated first-pass absorption for $(a)$ and $(b)$ and $62 \%$ for $(c)$ (multiple-pass absorption near the centre is estimated to be negligible in this launching geometry). The effective toroidal injection angles for all launchers are in the range $+25-29^{\circ}$. The three cases are similar limited discharges with $I_{\mathrm{p}}=230-240 \mathrm{kA}, \bar{n}_{\mathrm{e}}=(1.5-1.7) \times 10^{19} \mathrm{~cm}^{-3}, \kappa_{\text {edge }}=1.5-1.6$.

These observations are exemplified by figure 2 . The HXR emissivity profiles are clearly very different from, and in particular much broader than, the power deposition profiles. Suprathermal electrons thus exist in regions in which the theoretical EC power deposition is negligible; thus, either the deposition profile is highly anomalous, i.e. departs greatly from the theoretical predictions, or a mechanism must exist for transporting fast electrons far from the deposition region. The former hypothesis is in disagreement with a large body of evidence showing that ray tracing accurately predicts the EC power deposition [24, 25, 29]. These data thus indicate that transport plays a significant role in determining the suprathermal electron distribution at toroidal injection angles $>20^{\circ}$ and power densities in excess of $2 \mathrm{MW} \mathrm{m}^{-3}$.

While the similarity of the emissivity profiles in figures $2(a)$ and $(b)$ appears to suggest a possible profile resilience scenario, figure $2(c)$ shows that when the EC waves are deposited at sufficiently large radii the central emissivity falls to zero.

It is important to note that the main features of the profiles, i.e. their widths and the locations of the main peaks, are not dependent on the details of the profile inversion, and can be readily identified directly in the line-integrated profiles, as shown in figure 3 . While minor features of the inverted profiles, particularly the secondary peaks or plateaux, are in some cases not significant within the statistical uncertainties (shown on the integrated profiles), emissivity distributions as narrow as the EC power deposition profiles would clearly be incompatible with the measured line-integrated profiles.

The dynamics of suprathermal electrons are governed by several competing diffusive processes: collisional relaxation 


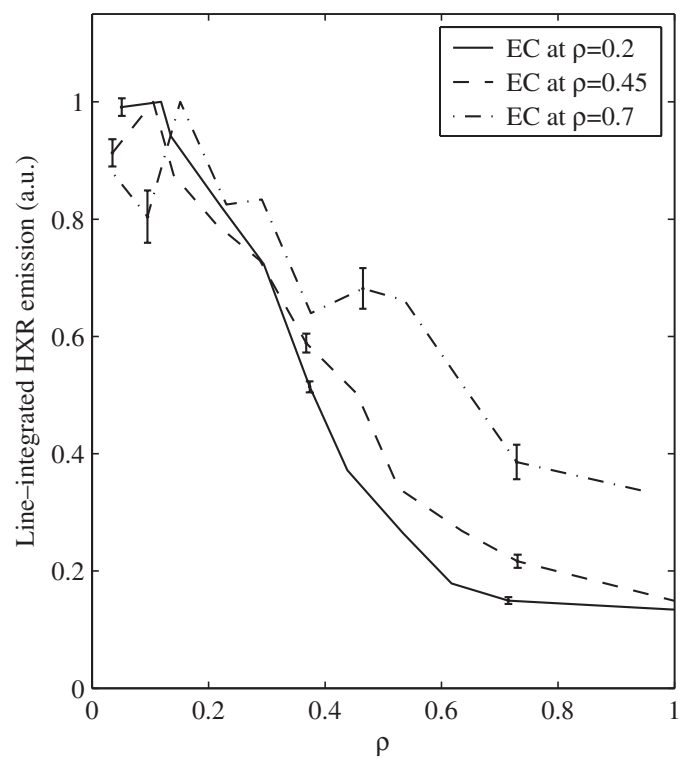

Figure 3. Line-integrated HXR emission profiles in the energy range 40-50 keV for TCV discharges 21982 (— $(-21991$ (- - - ) and $22003(-\cdot-)$, averaged over $0.45 \mathrm{~s}$, as a function of the minimum normalized radius for each chord, i.e. the point of tangency to the flux surface. The local emissivity profiles shown in figure 2 are derived from these data.

in velocity space (slowing-down and pitch-angle scattering), quasilinear rf diffusion in velocity space and anomalous turbulence-driven transport in real space. There is no 'source', since the heating process itself is of a diffusive nature (albeit not energy conserving). In general, the dynamics will be dominated by the shortest relaxation time in the system. To illustrate the potential effect of radial transport in TCV, let us adopt the common assumption that the relevant timescale is the collisional slowing-down time, $\tau_{\mathrm{SD}}$; in this case the effect of a radial diffusion coefficient, $D$, will be to broaden the fast electron profile by

$$
\Delta w \sim\left(D \tau_{\mathrm{SD}}\right)^{1 / 2}
$$

As an example, with typical central parameters for TCV discharges with ECCD $\left(n_{\mathrm{e}}=2 \times 10^{19} \mathrm{~m}^{-3}, T_{\mathrm{e}}=5 \mathrm{keV}\right.$, effective ion charge $Z_{\text {eff }}=4$ ), a diffusivity of the order of the bulk thermal diffusivity, $D \sim 3 \mathrm{~m}^{2} \mathrm{~s}^{-1}$, would broaden a $120 \mathrm{keV}$ population profile in TCV plasmas by $12 \mathrm{~cm}$, i.e. one-half the minor radius. Clearly, identical parameters would produce a less noticeable effect in a larger tokamak. It must be noted, however, that $D$ has generally been estimated to be between 0.3 and $1.0 \mathrm{~m}^{2} \mathrm{~s}^{-1}$ for the suprathermals sustained by lower hybrid current drive, with the exception of JET (where an estimate of $D \sim 6-10 \mathrm{~m}^{2} \mathrm{~s}^{-1}$ was given) [30].

A direct experimental measurement of the rate of transport of suprathermal electrons presents formidable difficulties, as was already documented in the long history of lower hybrid current drive experiments [30-33]. Some related studies with EC waves were reported in $[34,35]$. Since several potentially overlapping timescales are at play, an unambiguous determination of the complete system dynamics is difficult to conceive under controlled experimental conditions. On TCV, we have adopted the approach of applying multiple techniques

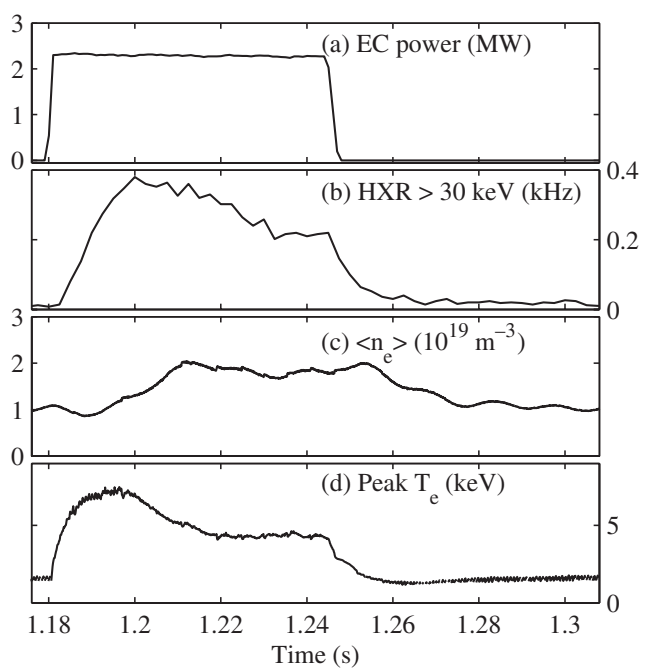

Figure 4. TCV shot 21978 (limiter configuration, $I_{\mathrm{p}}=240 \mathrm{kA}$, $\left.\kappa_{\text {edge }}=1.5-1.6\right)$ : time histories, during one EC modulation period $\left(\Phi=+25^{\circ}\right)$, of $(a)$ EC power, (b) HXR emission along a central chord for photon energies larger than $30 \mathrm{keV},(c)$ line-averaged electron density and $(d)$ peak electron temperature.

aimed at providing progressively stronger constraints on these dynamics.

Two such techniques have been employed recently to exploit the specific characteristics of the HXR and ECE diagnostics, respectively. Square-wave modulation of the electron cyclotron power has been performed in order to enhance the photon statistics and thus the temporal resolution of the HXR camera by coherent averaging. Photon statistics also dictated high power levels (typically $2.25 \mathrm{MW}$ with $50 \%$ duty cycle), which resulted in strong modulation of the bulk plasma parameters, particularly the density and the temperature (see figure 4). Since the electron energy confinement time is of the same order as the slowing-down time for electrons travelling at five to eight times the thermal velocity, a separation of the relevant timescales becomes impossible.

However, information on the suprathermal dynamics is contained in the temporal evolution of the spectral distribution of the HXR signals. If the slowing-down time is much shorter than the characteristic diffusion time, the spectra must be determined by local properties, with no effective communication over distant regions in space. Heating is applied to different velocity classes in different spatial regions, because of the Doppler shift needed to match the resonance condition locally. Additionally, the physical parameters that govern the steady-state distribution function, i.e. the EC power density, the bulk plasma density and the bulk electron temperature, are all spatially varying functions. Therefore the resulting spectral distribution should not be spatially uniform in general. However, in the relaxed state we invariably find that the spectral shape is essentially constant in space, even well outside the theoretical deposition region (albeit at a much lower amplitude) [36]; this is most naturally interpreted as an equilibration resulting from radial transport, but could also indicate an anomalous deposition profile in both physical and velocity space.

These dynamical measurements have shown for the first time that this uniformity is not present immediately after 

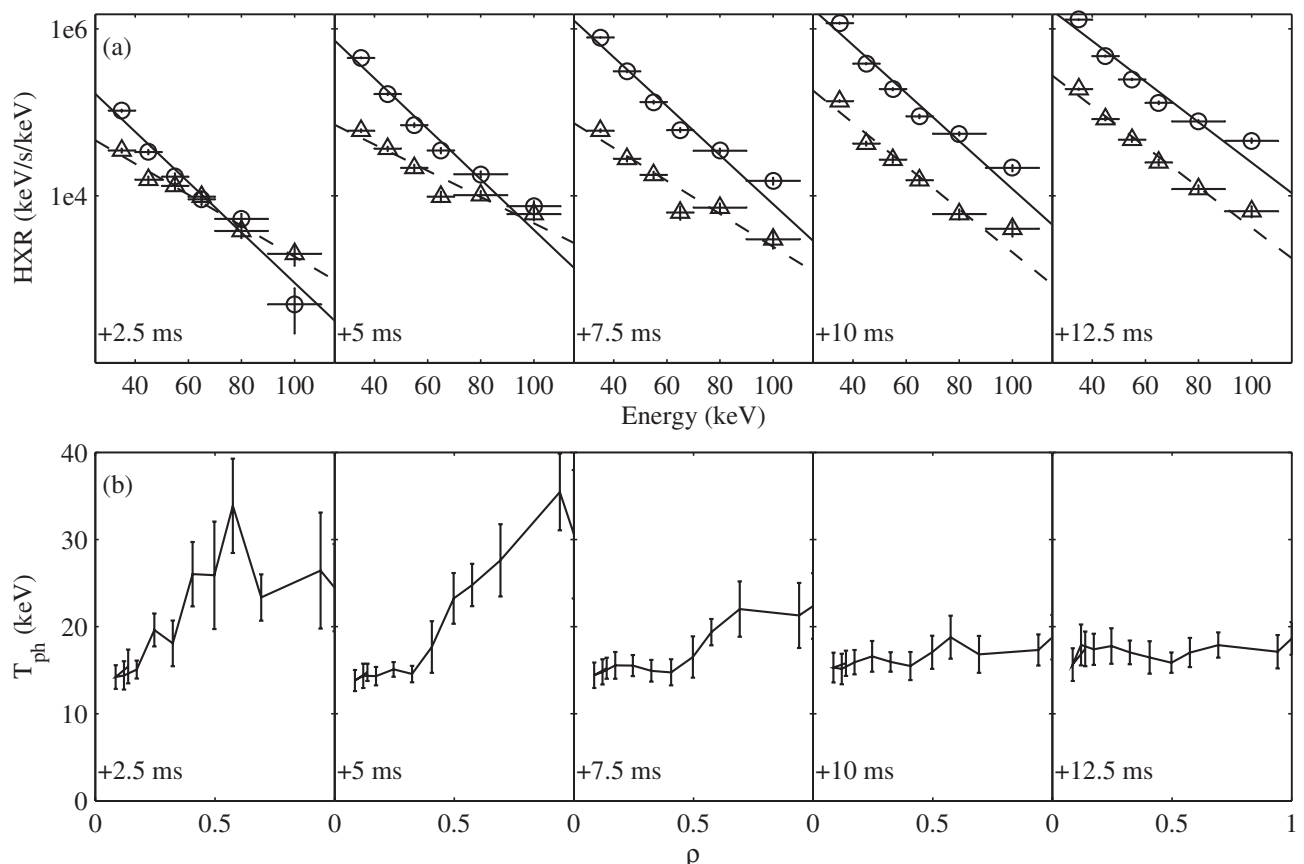

Figure 5. TCV shot 21978 (cf figure 4): (a) HXR emission (in units of power per photon energy interval) as a function of energy in five snapshots after the ECCD switch-on $(t=0)$, for a central $(-\odot)$ and an off-axis $\left(--^{-}-, \rho \sim 0.6\right)$ chord; $(b)$ spatial profiles of the photon temperature for the same snapshots ( $\rho$ here indicates the minimum normalized radius for each chord, i.e. the point of tangency to the flux surface). Data are coherently averaged over 13 modulation periods [19].

switch-on: as shown in figure 5(a) for a case with central co-ECCD $\left(\Phi=+25^{\circ}\right)$, the signal from a central chord is initially larger than the off-axis signal at low energy, while their roles are reversed at high energy. The on-axis spectrum does not change appreciably over time, whereas the offaxis spectrum becomes similar to the former over a period of approximately $10 \mathrm{~ms}$. A corresponding relaxation of the photon temperature profile towards a flat profile is seen in figure $5(b)$ [19]. If we assume that the relaxation is of a diffusive nature, we can deduce from the observed relaxation time a lower bound $\sim 1.5 \mathrm{~m}^{2} \mathrm{~s}^{-1}$ for the diffusivity in this discharge.

The higher sensitivity of the ECE system is compatible with perturbative studies. In a second experiment, we have applied short periodic ECCD pulses to the plasma and studied the ECE response by coherent averaging. Pulses longer than $0.3 \mathrm{~ms}$ were seen to result in a first peak, followed by a descent and a further increase until the end of the pulse, indicating that the pulse length exceeded the characteristic rf diffusion time. To avoid this complicating effect, the pulse was thus kept to a length of $0.25-0.3 \mathrm{~ms}$. The applied power was $0.45-0.9 \mathrm{MW}$ with a period of $10 \mathrm{~ms}$, and therefore a $3 \%$ duty cycle and an average power $<27 \mathrm{~kW}$, too low to affect the plasma parameters measurably.

Two examples, both with central ECCD, are shown in figure 6, with the plasma and ECE radiometer geometries shown in figure 7. In the case of figure 7(a) the ECE chord traverses the plasma centre, whereas in the case of figure 7(b) the smallest normalized minor radius accessed by ECE is approximately 0.55 . In both cases, the time to peak increases with the ECE frequency, as shown in figure 8. Moreover, the time lags are clearly larger when the ECE radiometer is aimed

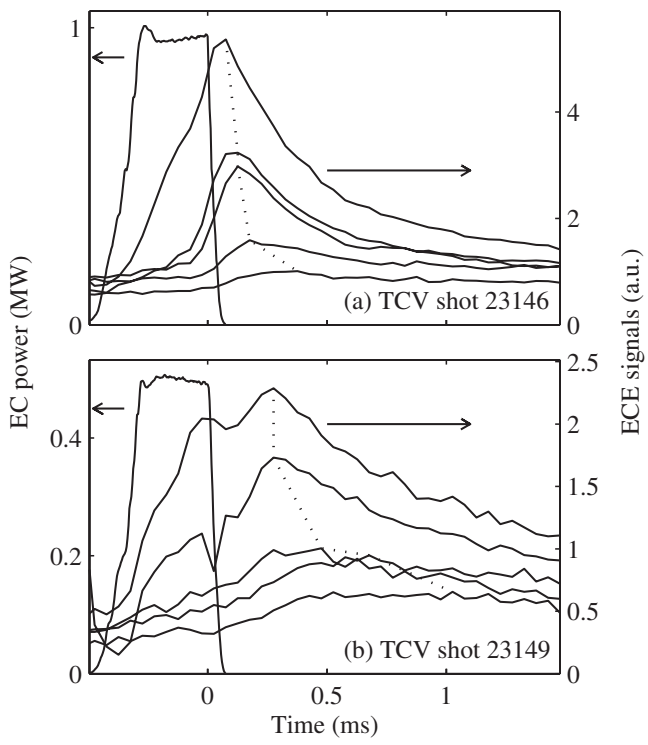

Figure 6. Response of selected ECE signals to a short central ECCD pulse (ending at $t=0$ ), averaged over 170 coherent pulses; the ECE frequency increases as the peak moves to later times: $(a)$ ECE radiometer on the midplane and $(b)$ ECE radiometer off-axis $(\rho>0.55)$ (see geometry in figure 7). The plasma conditions are similar in the two discharges: limiter configuration, $I_{\mathrm{p}}=240 \mathrm{kA}$, $\bar{n}_{\mathrm{e}}=(1.7-1.8) \times 10^{19} \mathrm{~cm}^{-3}, T_{\mathrm{e}, \max }=1.6-1.7 \mathrm{keV}, \kappa_{\text {edge }}=1.4-1.5$. The EC power in case $(a)$ is twice the power in case $(b)$.

off-axis: indeed, when plotted versus the cold-resonance $\rho$, the two curves connect smoothly to each other (figure 8(II)).

The cold-resonance radial coordinate in figure $8(I I)$ is to be taken only as a guiding parameter: indeed, in the presence of a substantial suprathermal tail, an increase in frequency can be associated either with a shift towards the high-field side 


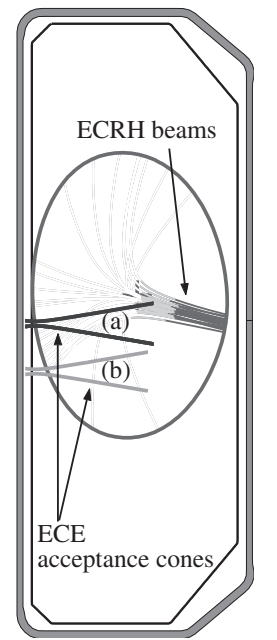

Figure 7. Geometries of ECRH launching and ECE radiometry for the two cases shown in figure 6 (in reality the radiometer is fixed and the plasma and EC beams are moved). The lighter grey portion of the beams denotes the region of $99 \%$ absorption.

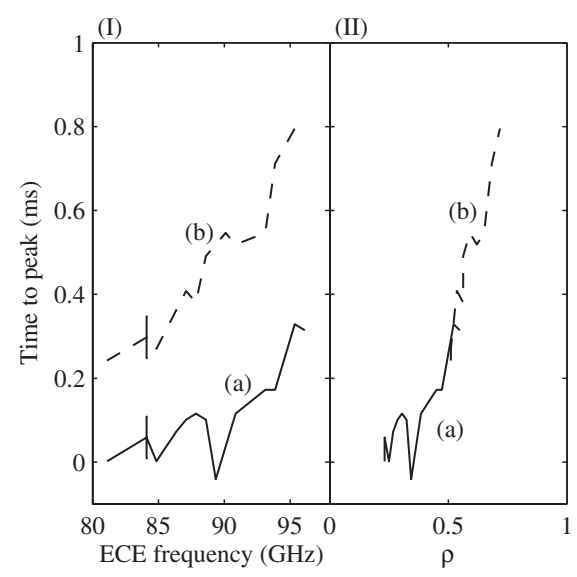

Figure 8. Time lag from the end of the ECCD pulse to the ECE peak, for the two cases (a) and (b) shown in figure 6: $(I)$ as a function of ECE frequency and (II) as a function of the equivalent $\rho$ at the cold resonance.

(larger minor radius) or with an increase in electron energy. However, for equal energy and frequency, the signals from the two chords originate in different spatial regions. Thus this observation corroborates the hypothesis that a transport mechanism is at play and that a non-negligible fraction of the fast electrons generated by ECCD is transported far from the deposition region. A more quantitative analysis will require modelling. In particular, the time lag will generally depend on both the slowing-down time and the diffusion time: therefore the detailed dependence of the time lag on the frequency cannot uniquely provide quantitative information on the diffusion coefficient. The time to peak is, however, only one parameter of the dynamical response. The full dynamics, particularly the decay time, can provide strong constraints on the modelling. Further work is planned in this direction.

\subsection{Quasilinear Fokker-Planck modelling}

The discrepancies between theory and experiment on the ECCD efficiency, mentioned at the beginning of this section,

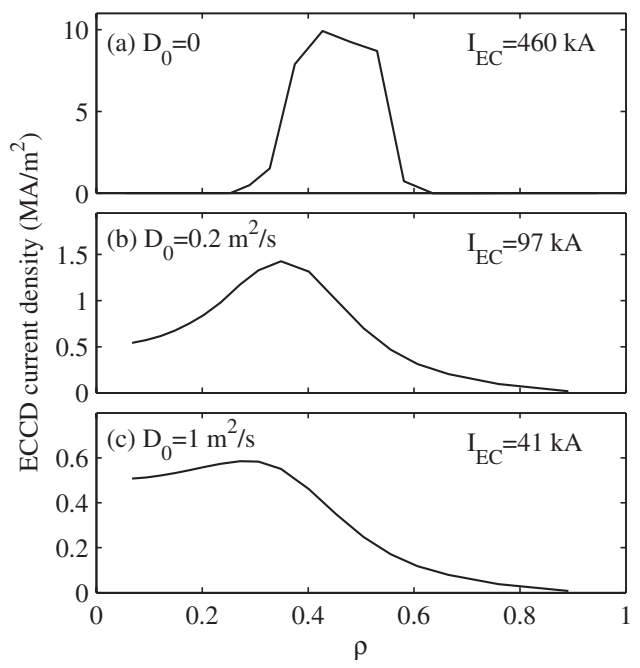

Figure 9. (a)-(c) EC-driven parallel current density profiles calculated by CQL3D for TCV discharge 21657 at $1.8 \mathrm{~s}$ (limiter configuration, $I_{\mathrm{p}}=72 \mathrm{kA}, \bar{n}_{\mathrm{e}}=5.1 \times 10^{18} \mathrm{~cm}^{-3}, T_{\mathrm{e}, \max }=3.3 \mathrm{keV}$, $\kappa_{\text {edge }}=1.6, Z_{\text {eff }}=3.5$ ), fully sustained by $0.9 \mathrm{MW}$ off-axis co-ECCD $\left(\Phi=+21^{\circ}\right)$, for three values of the central diffusivity, $D_{0}$ $\left(0,0.2,1 \mathrm{~m}^{2} \mathrm{~s}^{-1}\right)$, yielding different values of the EC-driven current (respectively, $460 \mathrm{kA}, 97 \mathrm{kA}, 41 \mathrm{kA}$ ); the current in case (c) matches the measurement at the low limit of the experimental uncertainty, since the bootstrap current, $I_{\mathrm{p}}-I_{\mathrm{EC}}$, is $23 \pm 8 \mathrm{kA}$. Linear calculations yield a driven current of $17 \mathrm{kA}$. The power deposition profile is similar to the calculated current profile in $(a)$ and peaks at $\rho=0.4$. A radial L-mode-like dependence is assumed for $D$, yielding a local diffusivity of $1.4 \mathrm{~m}^{2} \mathrm{~s}^{-1}$ in case $(c)$ at the deposition location; $D$ is assumed to be independent of velocity.

have been resolved by adjusting the diffusion coefficient in the Fokker-Planck code CQL3D, with optional dependences on the radial location and on the parallel velocity [15]. When $D$ is of the order of the bulk thermal diffusivity, the distribution of current-carrying fast electrons is broadened and non-linear enhancement is strongly inhibited; with $D$ in the range $0.5-5 \mathrm{~m}^{2} \mathrm{~s}^{-1}$, depending on the discharge and heating conditions, the experimentally measured EC-driven current can generally be reproduced (note that a comparison of the current density profile is not possible at present since no measurement is currently available on TCV). The same level of diffusivity does not change the predicted efficiency significantly in experimental situations in which non-linear enhancement is not expected [15]. Thus a unifying picture is beginning to emerge.

The achievement of fully non-inductive discharges driven entirely by off-axis ECCD and bootstrap currents [5, 20, 21] is a striking illustration of the effect of this suprathermal transport. The value of $D$ in the centre $\left(D_{0}\right)$ having been set such as to match the total driven current, the EC-driven current profile calculated by CQL3D is only slightly hollow in spite of the power being deposited approximately at halfradius, as illustrated by figure 9 . (It should be noted that the total current profile is nevertheless calculated to be considerably more hollow, owing to a large off-axis bootstrap current contribution.) Within the experimental uncertainties, an acceptable match is obtained with $D_{0}$ in the range $0.5-1.0 \mathrm{~m}^{2} \mathrm{~s}^{-1}$. In this case, $D$ has been taken to be independent of the parallel velocity and to increase with radius according to L-mode scaling [15]; in particular, at 


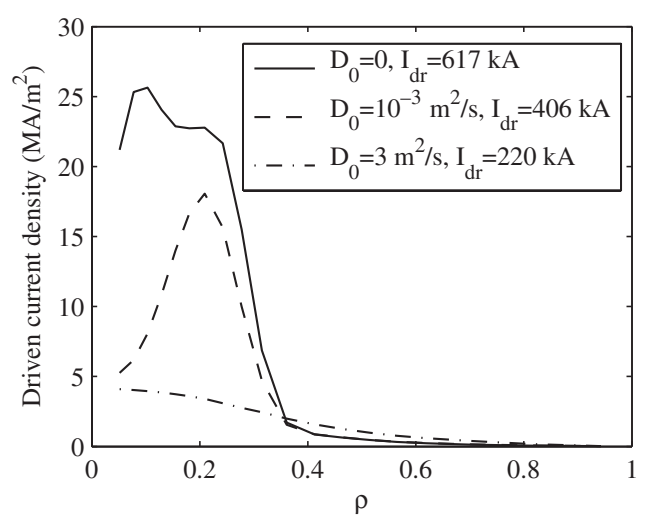

Figure 10. Externally driven $(\mathrm{EC}+\mathrm{Ohmic})$ parallel current density profiles calculated by CQL3D for the centrally heated TCV discharge 21982 at $1.0 \mathrm{~s}$ (limiter configuration, $I_{\mathrm{p}}=240 \mathrm{kA}$, $\bar{n}_{\mathrm{e}}=1.5 \times 10^{19} \mathrm{~cm}^{-3}, T_{\mathrm{e}, \max }=3.5 \mathrm{keV}, \kappa_{\text {edge }}=1.5 ; \mathrm{EC}$ power $=1.18 \mathrm{MW}, \Phi=+25^{\circ}$ ), for three values of the central diffusivity, $D_{0}\left(0, \longrightarrow ; 0.001 \mathrm{~m}^{2} \mathrm{~s}^{-1},-\cdots-; 3 \mathrm{~m}^{2} \mathrm{~s}^{-1}, \ldots \cdot-\right)$, yielding different values of the total current $(617,406,220 \mathrm{kA})$; the current in the last case matches the experimental measurement of the total plasma current. Within the experimental uncertainties, values of $D_{0}$ between 1.5 and $5.0 \mathrm{~m}^{2} \mathrm{~s}^{-1}$ yield acceptable results. The power deposition profile is similar to the calculated current profile in the $D_{0}=0$ case, and $D$ is assumed to be independent of velocity.

the EC power deposition location, $D$ equals approximately $0.7-1.4 \mathrm{~m}^{2} \mathrm{~s}^{-1}$. It is important to note that even in the presence of this radial diffusion, the total driven current $(49 \pm 8 \mathrm{kA})$ remains substantially larger than that predicted by linear theory (17 kA).

When the loop voltage is non-zero, CQL3D calculates the sum of the Ohmic and EC-driven currents self-consistently, taking into account the effect of the electric field. This current is then compared with the measured total current minus the bootstrap current (calculated from pressure profile measurements by Thomson scattering). This procedure is hampered by limited accuracy in the Ohmic component, which can be ascribed in particular to uncertainties in $Z_{\text {eff }}$ [37]. For this reason, fully non-inductive discharges in which the Ohmic current is zero provide an especially strong constraint to the modelling. Nevertheless, the need for a finite diffusion coefficient is readily apparent also in discharges with a nonzero loop voltage, as shown in figure 10 for a centrally heated case. In the absence of diffusion, the overestimation of the total current far exceeds the uncertainty in the modelling.

To gain further insight into the physics of ECCD at the high power densities of $\mathrm{TCV}$, it is instructive to compare the calculated parallel current density distributions in velocity space with and without diffusion. The differential densities $\mathrm{d} j / \mathrm{d}\left|v_{\|}\right|$and $\mathrm{d} j / \mathrm{d} v_{\perp}$ (where $\|$ and $\perp$ are relative to the total magnetic field) are plotted in figure 11 for the same noninductive discharge of figure 9 at the power deposition location $(\rho=0.4)$. Here,

$$
\frac{\mathrm{d} j}{\mathrm{~d}\left|v_{\|}\right|}=-e\left|v_{\|}\right| \int_{0}^{\infty} 2 \pi\left[f\left(v_{\perp},\left|v_{\|}\right|\right)-f\left(v_{\perp},-\left|v_{\|}\right|\right)\right] v_{\perp} \mathrm{d} v_{\perp}
$$

and

$$
\frac{\mathrm{d} j}{\mathrm{~d} v_{\perp}}=-2 \pi e v_{\perp} \int_{-\infty}^{\infty} f\left(v_{\perp}, v_{\|}\right) v_{\|} \mathrm{d} v_{\|},
$$

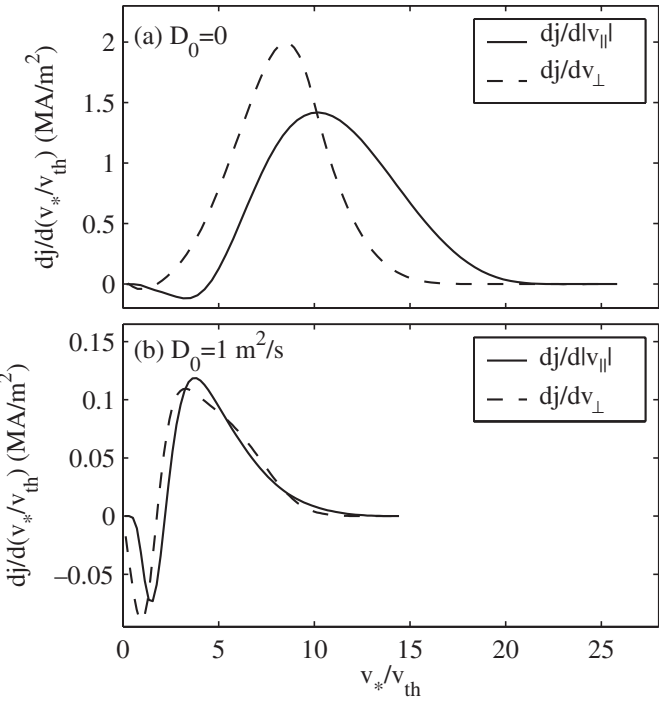

Figure 11. Differential parallel current densities in velocity space, $\mathrm{d} j / \mathrm{d}\left|v_{\|}\right|(-)$and $\mathrm{d} j / \mathrm{d} v_{\perp}(---)$, calculated by CQL3D for TCV shot 21657 at $\rho=0.4$ and $1.8 \mathrm{~s}$ (cf figure 9): (a) $D_{0}=0$ and (b) $D_{0}=1 \mathrm{~m}^{2} \mathrm{~s}^{-1}$. In the abscissa $v_{*}$ denotes $\left|v_{\|}\right|$and $v_{\perp}$, respectively, for $\mathrm{d} j / \mathrm{d}\left|v_{\|}\right|$and $\mathrm{d} j / \mathrm{d} v_{\perp}$.

where $f\left(v_{\perp}, v_{\|}\right)$is the electron distribution function. The average energy of the current-carrying electrons decreases from $\sim 70-100$ times the thermal energy in the case of no diffusion to $\sim 10-20$ times the thermal energy with $D_{0}=$ $1 \mathrm{~m}^{2} \mathrm{~s}^{-1}$.

The effectiveness of diffusion in broadening the spatial distribution of fast electrons is enhanced at the high energies to which these electrons are accelerated by the high-power EC waves: since the slowing-down time increases with velocity as $v^{3}$, the broadening, $\Delta w$, according to equation (1), increases as $v^{3 / 2}$. By applying equation (1) to the discharge of figure 11, with parameters $n_{\mathrm{e}}=6 \times 10^{18} \mathrm{~m}^{-3}, T_{\mathrm{e}}=1.6 \mathrm{keV}, Z_{\text {eff }}=3$, $D=1 \mathrm{~m}^{2} \mathrm{~s}^{-1}$ at the deposition location, an electron energy of $80 \times T_{\mathrm{e}}=130 \mathrm{keV}$ results in $\Delta w \simeq 14 \mathrm{~cm}$, i.e. a broadening of over one-half the radius of the plasma column. This is of course only an approximate upper bound: since transport itself acts to reduce the average electron energies, as demonstrated by figure 11, the width of the relaxed fast electron profile will be somewhat smaller than that given by this estimate. However, this example serves to illustrate the important concept that the ECCD spatial broadening increases inherently with power simply by virtue of the longer lifetimes of the current carriers, even in the absence of any direct power degradation, i.e. even if $D$ is independent of power.

Longer lifetimes also imply a reduction in current drive efficiency owing to fast particles escaping from the plasma column [38]. Note however that this efficiency loss (as opposed to mere broadening of the driven current profile) becomes significant only when $\Delta w$ becomes comparable with the plasma radius (see also [39]), and would be considerably overestimated by replacing the plasma radius with the ECRH deposition width as the characteristic scale length, as proposed in [29]. In TCV the primary effect of radial transport is to regulate the electron distribution function and thus the ECCD efficiency at an intermediate level between the linear and quasilinear limits, as well as to broaden the current drive 

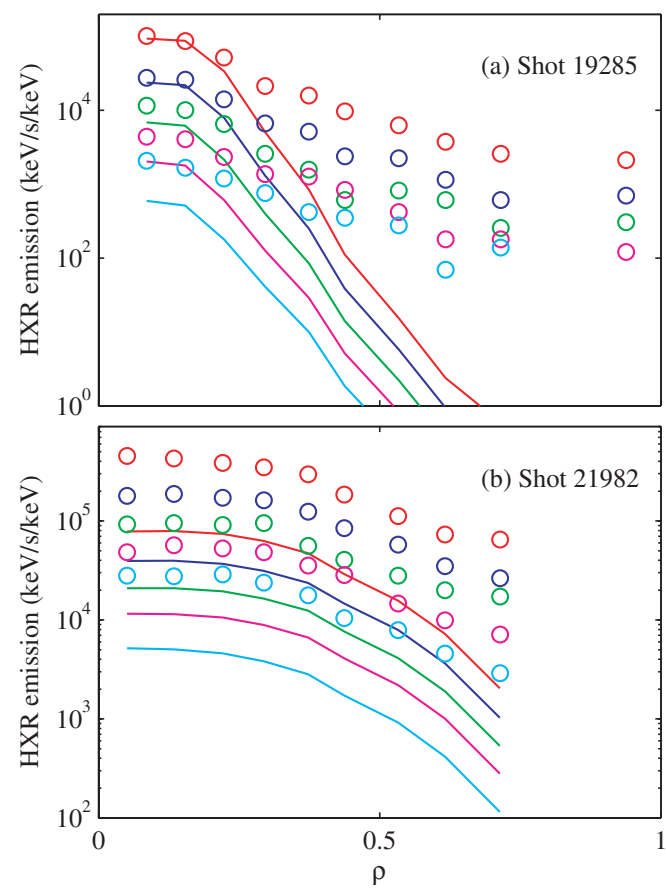

Figure 12. (a) TCV shot 19285 (0.45 MW central co-ECCD, $\Phi=+13^{\circ}$; limiter configuration, $I_{\mathrm{p}}=205 \mathrm{kA}$, $\left.\bar{n}_{\mathrm{e}}=(1.8-1.9) \times 10^{19} \mathrm{~cm}^{-3}, T_{\mathrm{e}, \max }=3 \mathrm{keV}, \kappa_{\text {edge }}=1.3\right):$ measured HXR emission averaged from 0.3 to $0.5 \mathrm{~s}$ (circles) versus that predicted by CQL3D at $0.45 \mathrm{~s}$ (curves), as a function of chordal spatial location (minimum normalized radius) for five energy channels of $8 \mathrm{keV}$ width in the $16-56 \mathrm{keV}$ range (descending amplitude for increasing energy); the value of $D_{0}$ is adjusted in CQL3D to reproduce the experimental current and is equal to $0.1 \mathrm{~m}^{2} \mathrm{~s}^{-1} ; D$ is assumed to be independent of velocity and $(b) \mathrm{TCV}$ shot $21982\left(1.18 \mathrm{MW}\right.$ central co-ECCD, $\Phi=+25^{\circ}$; limiter configuration, $I_{\mathrm{p}}=240 \mathrm{kA}, \bar{n}_{\mathrm{e}}=1.5 \times 10^{19} \mathrm{~cm}^{-3}$,

$T_{\mathrm{e}, \max }=3.5 \mathrm{keV}, \kappa_{\text {edge }}=1.5$; cf figure 10 ): experimental average from 0.65 to $1.35 \mathrm{~s}$, modelling for $1.0 \mathrm{~s}$, energy channels $30-40$, $40-50,50-60,60-70$ and $70-90 \mathrm{keV} ; D_{0}=3 \mathrm{~m}^{2} \mathrm{~s}^{-1}$.

profile, whereas global fast electron losses do not appear to be significant.

It should be further noted that in a future reactor-scale tokamak such as ITER the large machine size would result both in a reduced power density and in an increased device scale length, rendering the spatial broadening negligible for all practical purposes.

Different forms of functional dependence of the diffusivity on the parallel velocity, derived from different models of electrostatic or magnetic turbulence, have been employed in CQL3D, with no conclusive evidence thus far in favour of one or the other [37]. The reason for this can be gleaned from figure 11: different models will give similar results, provided the value of the diffusivity is approximately the same in the current-carrying region of velocity space.

A remarkable similarity is observed between the current distributions in the parallel and perpendicular velocities. On the low-field side of the nominal resonance, electrons that resonate with EC waves of finite parallel wave number possess suprathermal parallel velocities; the wave-particle interaction, on the other hand, acts to increase the perpendicular velocity. The simulations show that the combination of the two effects results in a current distribution that is approximately symmetric
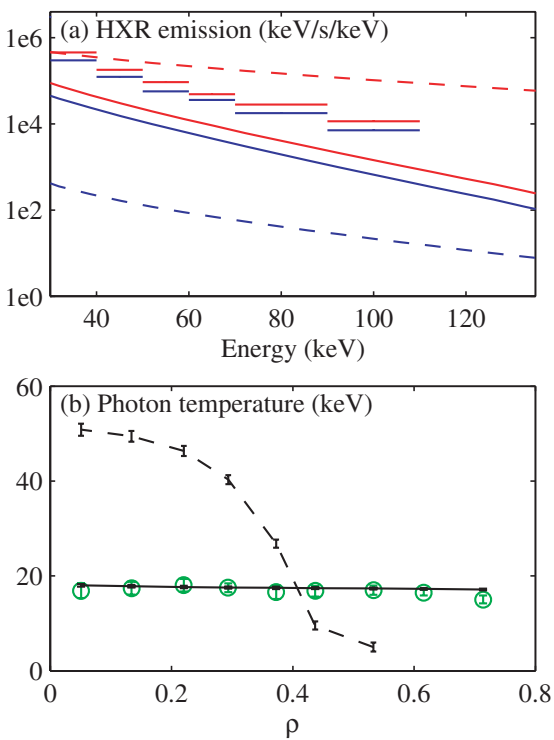

Figure 13. TCV shot 21982 (cf figure 10): (a) HXR emission spectrum for a central chord (red) and a chord with a minimum $\rho \sim 0.37$ (blue): experimental data averaged from 0.65 to $1.35 \mathrm{~s}$ (horizontal bars), CQL3D calculations at $1.0 \mathrm{~s}$ with diffusivity $D_{0}=3 \mathrm{~m}^{2} \mathrm{~s}^{-1}\left(-\right.$ ) and $D_{0}=0(---)$ and $(b)$ photon temperature calculated from an exponential fit to the spectra in the interval $30-120 \mathrm{keV}$, as a function of radial position $\rho$ (minimum normalized radius for each chord): experimental data (green circles), CQL3D data with $D_{0}=3 \mathrm{~m}^{2} \mathrm{~s}^{-1}$ (—) and $\left.D_{0}=0(--)^{-}\right)$.

in $\left|v_{\|}\right|$and $v_{\perp}$ (a considerably weaker symmetry, however, than complete pitch-angle isotropy, which is certainly not satisfied).

\subsection{Comparison of calculated and measured HXR emission}

A further approach to studying the suprathermal electron relaxation phenomena consists of direct comparisons of HXR data with the bremsstrahlung emission predicted by the CQL3D code for the specific geometry of our diagnostic. The results of these comparisons have been mixed. Good agreement is generally found in the spectral distribution, i.e. in the photon temperature (see figure 12). The absolute emission in the deposition region is often well reproduced (see figure 12(a)), although it is underestimated in some cases by up to an order of magnitude [15, 19, 37, 40] (figure 12(b)). The emission profile shape is found to be in good agreement in higher-power cases with $D_{0}>0.5 \mathrm{~m}^{2} \mathrm{~s}^{-1}$ (figure $12(b)$ ), whereas in the lower-power, centrally heated case of figure $12(a)$, the low diffusivity $\left(D_{0}=0.1 \mathrm{~m}^{2} \mathrm{~s}^{-1}\right)$ suggested by the modelling leads to an emission profile that is considerably more peaked than its experimental counterpart. Preliminary results indicate that the match can be improved in this case by adjusting the dependence of the diffusivity on the parallel electron velocity. These comparisons could thus ultimately provide stronger constraints for the transport modelling.

In the higher-power scenarios, without radial transport not only is the predicted current much too large, the predicted HXR signal is also far narrower spatially than the measured one, as shown in figure 13(a). In particular, these results provide strong evidence that the spatial uniformity of the spectral shape is a consequence of spatial transport. The 
shape is parametrized in figure $13(b)$ by a photon temperature, which is seen to be a strong function of minor radius in the case $D_{0}=0$, whereas with $D_{0}=3 \mathrm{~m}^{2} \mathrm{~s}^{-1}$ a uniform temperature is recovered, which is moreover in excellent quantitative agreement with the experimental values. Note that in the diffusionless case the calculated photon temperature appears to decrease with radius, whereas the opposite behaviour is observed experimentally immediately after power switch-on (see figure 5). This disagreement is not significant per se, since the CQL3D calculations refer to steady-state conditions and cannot reproduce a dynamical evolution in which strong self-inductive transient electric fields are present. In addition, the calculated spectra are only approximately exponential; in particular, at a higher energy the slope becomes less steep (higher temperature) at larger radii. The key observation in both the experiment (initial spectrum before diffusion has occurred) and the simulation (steady-state scenario without diffusion) is that the shape of the spectrum is not uniform in space, since all the physical quantities that determine it are indeed not uniform.

\section{Conclusions and outlook}

ECCD is being used in TCV as a powerful tool for current and pressure profile shaping, especially with the high degree of control afforded by fully non-inductive operation, and thus in the absence of an electric field, in steady state. At the same time, TCV is one of the premier test beds for fundamental inquiries into the physics of ECCD and the associated suprathermal electron dynamics, owing to a uniquely powerful and flexible ECRH system and dedicated diagnostics, complemented by an advanced and comprehensive Fokker-Planck quasilinear code. The fundamental relaxation processes governing the suprathermal dynamics are being investigated through multiple experimental approaches and comparisons with modelling.

Strong evidence supports the conclusion that the suprathermal electrons excited by ECCD undergo cross-field transport at a rate comparable with that of thermal transport, resulting in a reduction of the non-linear effects expected at the TCV power densities, and particularly of the attendant nonlinear enhancement of the current drive efficiency. However, in spite of this radial transport the efficiency still exceeds that predicted by linear theory, which has been shown to be fairly accurate at lower power levels [41]. A gain in efficiency is therefore still obtained by operating at higher power levels.

It should also be stressed that the observed broadening of the suprathermal current carrier distribution does not affect the applicability of ECRH as a tool for pressure profile shaping and, through modification of the conductivity, for current profile shaping, since the power deposition remains very localized. In applications with characteristic timescales exceeding the energy confinement time, the shape of the pressure profile is primarily controlled by thermal energy transport rather than by the breadth of the power deposition profile. Transport of suprathermals, when they are present (i.e. in an ECCD configuration), occurs at a rate close to that of thermal energy and thus does not alter this picture.

By contrast, the localization of current drive is, of course, significantly diminished in the high power density scenario of
TCV. However, even under these conditions the global current profile can be controlled to a considerable extent by moving the centroid of each EC source's deposition profile, with dramatic effects on the energy confinement and on the bootstrap current fraction $[5,20,21]$. In addition, the broadening of the ECCD profile at the very small parallel wave numbers used, e.g. in sawtooth control experiments [42], is expected to be modest as a result of the lower electron energies involved [36] (through the aforementioned $\Delta w \propto v^{3 / 2}$ dependence).

Much remains to be known about the underlying transport mechanism. Fokker-Planck simulations using different transport models, based on both electrostatic and electromagnetic turbulence, have thus far been inconclusive. Stronger constraints are however expected to be provided by further comparisons between the calculated and measured HXR emission. A crucial question that must be addressed is the dependence of this transport on the ECRH power density itself; it is plausible for instance to suppose a power degradation mechanism to be at play as in thermal transport, as suggested also by the modelling results discussed in this paper, but this hypothesis has not yet been tested systematically.

\section{Acknowledgments}

The authors are grateful to the entire TCV team for the operation of the tokamak and of the auxiliary heating systems, and to CEA-Cadarache for the loan of the HXR camera and associated equipment. It is a pleasure to acknowledge the continuing assistance of R.W. Harvey in the operation of the CQL3D code. This work was supported in part by the Swiss National Science Foundation.

\section{References}

[1] Erckmann V. and Gasparino V. 1994 Plasma Phys. Control. Fusion 361869

[2] Lloyd B. 1998 Plasma Phys. Control. Fusion 40 A119

[3] Alberti S. et al 2001 Fusion Eng. Des. 53387

[4] Piosczyk B. et al 2002 Proc. 19th Int. Conf. on Fusion Energy 2002 (Lyon, 2002) (Vienna: IAEA) CD-ROM file CT-7Rb and http://www.iaea.org/programmes/ripc/physics/fec2002/ $\mathrm{html} / \mathrm{fec} 2002 . \mathrm{htm}$

[5] Goodman T.P. et al 2003 An overview of recent results from the TCV tokamak Nucl. Fusion submitted

[6] Sauter O. et al 2000 Phys. Rev. Lett. 843322

[7] Coda S. et al 2000 Plasma Phys. Control. Fusion 42 B311

[8] Sauter O. et al 2001 Phys. Plasmas 82199

[9] Peysson Y. and Imbeaux F. 1999 Rev. Sci. Instrum. 703987

[10] Peysson Y., Coda S. and Imbeaux F. 2001 Nucl. Instrum. Methods Phys. Res. A $\mathbf{4 5 8} 269$

[11] Blanchard P., Alberti S., Coda S., Weisen H. and Klimanov I. 2002 Plasma Phys. Control. Fusion 442231

[12] TFR Group and Fidone I. 1981 Phys. Rev. A 242861

[13] Harvey R.W. and McCoy M.G. 1993 Proc. IAEA Technical Committee Meeting on Advances in Simulation and Modeling in Thermonuclear Plasmas (Montreal, 1992) (Vienna: IAEA) p 498

[14] Matsuda K. 1989 IEEE Trans. Plasma Sci. 176

[15] Harvey R.W., Sauter O., Prater R. and Nikkola P. 2002 Phys. Rev. Lett. 88205001

[16] Fisch N.J. and Boozer A.H. 1980 Phys. Rev. Lett. 45720

[17] Harvey R.W., McCoy M.G. and Kerbel G.D. 1989 Phys. Rev. Lett. 62426

[18] Coda S. et al 1999 Proc. 26th Eur. Conf. on Controlled Fusion and Plasma Physics (Maastricht, 1999) vol 23J (Europhys. Conf. Abstr.) (Geneva: European Physical Society) p 1097 
[19] Coda S. et al 2002 Proc. 29th Eur. Conf. on Controlled Fusion and Plasma Physics (Montreux 2002) vol 26B (Europhys. Conf. Abstr.) (Geneva: European Physical Society) O-4.03

[20] Sauter O. et al 2002 Proc. 29th Eur. Conf. on Controlled Fusion and Plasma Physics (Montreux, 2002) vol 26B (Europhys. Conf. Abstr.) (Geneva: European Physical Society) P-2.087

[21] Sauter O. et al 2002 Proc. 19th Int. Conf. on Fusion Energy 2002 (Lyon, 2002) (Vienna: IAEA) CD-ROM file EX/P5-06 and http://www.iaea.org/programmes/ripc/physics/fec2002/ html/fec2002.htm

[22] Pochelon A. et al 2002 Proc. 19th Int. Conf. on Fusion Energy 2002 (Lyon, 2002) (Vienna: IAEA) CD-ROM file EX/P5-14 and http://www.iaea.org/programmes/ripc/physics/fec2002/ html/fec2002.htm

[23] Alberti S. et al 2002 Nucl. Fusion 4242

[24] Goodman T.P. et al 1998 Proc. 2nd Europhysics Topical Conf. on Radio Frequency Heating and Current Drive of Fusion Devices (Brussels, 1998) p 245

[25] Petty C.C. et al 2000 Proc. 4th Int. Conf. on Strong Microwaves in Plasmas (Nizhny Novgorod, 1999) (Nizhny Novgorod: Russian Academy of Sciences) vol 1, p 41

[26] Petty C.C. et al 2001 Nucl. Fusion 41551

[27] Nikkola P. and Sauter O. 2000 Proc. Joint Varenna-Lausanne Int. Workshop (Varenna, 2000) (Bologna: Editrice Compositori) Theory Fusion Plasmas p 345
[28] Cohen R.H. 1987 Phys. Fluids 302442

[29] Luce T.C. 2002 IEEE Trans. Plasma Sci. 30734

[30] Peysson Y. 1993 Plasma Phys. Control. Fusion 35 B253

[31] Kirkwood R. et al 1990 Phys. Fluids B 21421

[32] Giruzzi G. et al 1995 Phys. Rev. Lett. 74550

[33] Jones S.E. et al 1995 Phys. Plasmas 21548

[34] Lopes Cardozo N.J. et al 1993 Proc. 14th Int. Conf. on Plasma Physics and Controlled Nuclear Fusion Research 1992 (Würzburg, 1992) vol 1 (Vienna: IAEA) p 271

[35] Esipchuk Yu.V., Kirneva N.A., Martynov A.A. and Trukhin V.M. 1995 Fizika Plazmy 21577

Esipchuk Yu.V., Kirneva N.A., Martynov A.A. and Trukhin V.M. 1995 Plasma Phys. Rep. 21577

[36] Coda S. et al 2001 Proc. 28th Eur. Conf. on Controlled Fusion and Plasma Physics (Madeira, 2001) vol 25A (Europhys. Conf. Abstr.) (Geneva: European Physical Society) p 301

[37] Nikkola P., Coda S., Harvey R.W. and Sauter O. 2002 Proc. Joint Varenna-Lausanne Int. Workshop (Varenna, 2002) (Bologna: Società Italiana di Fisica) Theory Fusion Plasmas p 315

[38] Luckhardt S.C. 1987 Nucl. Fusion 271914

[39] O’Brien M.R., Cox M. and McKenzie J.S. 1991 Nucl. Fusion 31583

[40] Nikkola P. et al 2003 Nucl. Fusion 431343

[41] Petty C.C. et al 2003 Nucl. Fusion $\mathbf{4 3} 700$

[42] Angioni C. et al 2003 Nucl. Fusion 43455 